\title{
Coupling Resistive Switching Devices with Neurons: State of the Art and Perspectives
}

\author{
Alessandro Chiolerio ${ }^{1 *}$, Michela Chiappalone ${ }^{2}$, Paolo Ariano ${ }^{1}$ and Sergio Bocchini ${ }^{1}$ \\ ${ }^{1}$ Center for Sustainable Future Technologies, Istituto Italiano di Tecnologia, Torino, Italy, ${ }^{2}$ Neuroscience and Brain \\ Technologies Department, Istituto Italiano di Tecnologia, Genova, Italy
}

\section{OPEN ACCESS}

Edited by:

Ramona Samba,

Natural and Medical Sciences Institute, Germany

Reviewed by: Alexander Dityatev,

German Center for Neurodegenerative Diseases (DZNE), Germany Stefano Vassanelli, University of Padua, Italy

*Correspondence: Alessandro Chiolerio alessandro.chiolerio@iit.it

Specialty section

This article was submitted to Neural Technology,

a section of the journal

Frontiers in Neuroscience

Received: 29 February 2016 Accepted: 31 January 2017 Published: 15 February 2017

Citation:

Chiolerio A, Chiappalone M, Ariano P and Bocchini S (2017) Coupling Resistive Switching Devices with Neurons: State of the Art and Perspectives. Front. Neurosci. 11:70. doi: 10.3389/fnins.2017.00070
Here we provide the state-of-the-art of bioelectronic interfacing between biological neuronal systems and artificial components, focusing the attention on the potentiality offered by intrinsically neuromorphic synthetic devices based on Resistive Switching (RS). Neuromorphic engineering is outside the scopes of this Perspective. Instead, our focus is on those materials and devices featuring genuine physical effects that could be sought as non-linearity, plasticity, excitation, and extinction which could be directly and more naturally coupled with living biological systems. In view of important applications, such as prosthetics and future life augmentation, a cybernetic parallelism is traced, between biological and artificial systems. We will discuss how such intrinsic features could reduce the complexity of conditioning networks for a more natural direct connection between biological and synthetic worlds. Putting together living systems with RS devices could represent a feasible though innovative perspective for the future of bionics.

Keywords: cybernetics, bio-electronic systems, resistive switching devices, memristors, neuromorphic devices, multielectrode arrays

\section{INTRODUCTION}

The brain is the most powerful and complex known computational system. A recent work evaluates the memory capacity of the human brain to be in the order of $10^{15}$ Bytes (Bartol et al., 2016). Unlike the hardware and software of a machine, the mind and brain are not distinct entities, feature that resembles the so called firmware. How could we represent a neuronal synapse, a complex structure containing hundreds of different proteins with a single line of code? We still do not know the detailed circuitry of any region of the brain well enough to reproduce its structure and, as a consequence, its behavior (Brooks et al., 2012).

The technological roadmap toward integration of synthetic and biological functions was described in the past as cybernetics, in a definition given by N. Wiener (Wiener, 1961) that

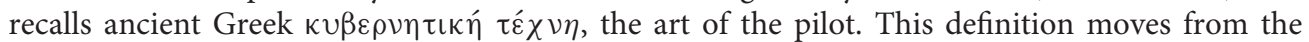
hypothesis that there is a substantial analogy between self-regulation mechanisms in living beings and machines, based on information flow and feedback/closed loop.

Putting the focus on silicon microdevices, we should trace a boundary between (Breslin and O’Lenskie, 2001).

(1) extrinsic neuromorphic systems, based on CMOS circuits that enable processing of information as occurs naturally in biological brains, including silicon based artificial synapses and artificial neurons sorted in neural networks, that are outside the scopes of this review; 
(2) intrinsic neuromorphic systems, artificial synapses or arrays of elements that inherently possess key figures such as plasticity, non-linearity, spiking processing capabilities.

Giant projects / frameworks, such as the DARPA SyNAPSE program (Systems of Neuromorphic Adaptive Plastic Scalable Electronics) and the EU Human Brain Project deal with standard extrinsic systems and therefore are outside the scopes of this Perspective. Their paradigm is reproducing the configurational complexity by emulating a simplified physical model in an extremely high number of elements (at least $10^{10}$ neurons and $10^{14}$ synapses).

\section{NEURONAL NETWORKS IN VITRO: A SIMPLIFIED MODEL OF BRAIN CIRCUITS}

The neuronal assembly, as defined by Hebb (1949) is "a group of cells that share similar static and dynamic response properties, constituting the simplest instance of a representative process." A network of sparsely coupled neurons from different brain areas developing in vitro is a useful experimental model for a generic assembly, since it has been proven to retain fundamental properties of the original tissue and the same distribution of cell types (Marom and Shahaf, 2002). Dissociated neurons can be cultured in vitro for many months (Potter and DeMarse, 2001) as they form a new 2D network functionally connected by synapses. This experimental model can be easily coupled to substrate-integrated Micro-Electrode Arrays (MEAs). Planar MEAs consists of glass or silicon over which a conductor is patterned in order to design specific layout of electrodes where electrogenic cells can grow and develop (Gross et al., 1977; Maher et al., 1999; Berdondini et al., 2009). They were first developed in the late 70's thanks to the advancements in micro-fabrication technologies. The lab of Prof. G. Gross at the University of North Texas has pioneered in the development of microelectrode arrays to be coupled to neuronal networks (Gross et al., 1977; Gramowski et al., 2000). Nowadays these devices allow in vitro (Jimbo and Kawana, 1992; Gross et al., 1995; Massobrio et al., 2015) and also in vivo (Vassanelli et al., 2012; Vassanelli, 2014) multi-site, long-term recordings of the activity of neuronal populations and extracellular stimulation from one or more electrodes of the array. Further, advancements of this technique allow nowadays recording and stimulating from hundreds/thousands of electrodes (Kaul et al., 2004; Heer et al., 2006; Pearce and Williams, 2007; Frey et al., 2009; Thewes et al., 2016), and simultaneously record electrical and optical signal through transparent diamond electrodes (Ariano et al., 2005, 2009). Multiparametric measurements are suitable for research devices and laboratory investigation while the biological electrical activity and MEA-like devices appear, at a glance, the most suitable technology that should be easily wearable and provide comfort in addition to functionality (Stoppa and Chiolerio, 2014).

Dissociated neurons in culture show spontaneous electrical activity that can be easily measured and evaluated through the use of MEAs. The firing rate of the cells changes during the in vitro development, related to the age of the network (Van Pelt et al., 2004; Bologna et al., 2010). Starting from the second week in culture, spikes tend to cluster into bursts, thus presenting a kind of activity which persists for the whole life span and represents a mature state of the network (Maeda et al., 1995; Bonifazi et al., 2005; Chiappalone et al., 2006, 2007; Eytan and Marom, 2006; Biffi et al., 2013; Bisio et al., 2014). The "bursting" mode of activity can be also modulated in vitro by appropriate electrical and/or chemical stimulation. In general, low frequency, sustained electrical stimulation locks the phase of periodic bursts to the applied stimuli (Maeda et al., 1995). Higher rates of stimulation induce a transition from synchronized bursting activity into a more sparse spiking behavior (Wagenaar et al., 2005). In particular, in vitro experiments in different neural preparations have shown that Hebbian plasticity, in the form of long-term potentiation and depression, provides the basis of many models of learning and memory (Shahaf and Marom, 2001; Chiappalone et al., 2008; Le Feber et al., 2010; Stegenga et al., 2010, and for a complete review see Massobrio et al., 2015).

For the above reasons, neuronal networks represent a very powerful yet simple and easy accessible system that retains important properties of the original brain tissue. Coupled to electronic devices that can read, process and stimulate their electrophysiological activity, they form the so called "bio-artificial living systems.” These innovative hybrid systems are now paving the way for next generation of neural interfaces and intelligent neurally-inspired information processing systems.

\section{HYBRID INTERFACES AND CLOSED-LOOP SYSTEMS: TOWARD BIO-ELECTRONIC COMPUTATIONAL SYSTEMS}

A hybrid system is defined as the combination between a biological and an artificial element (Figure 1A). Typically a biological element is able to "talk" to an artificial one thanks to specific algorithms that can translate the language of the cells into commands or instructions (Mussa-Ivaldi et al., 2010). In neuroscience in particular there has been a growing interest for "closed-loop" experiments, in which recordings of various types are used to modulate stimulation (Mavoori et al., 2005; Jackson et al., 2006; Venkatraman et al., 2009). The underlying rationale is that the dynamic and adaptive properties of neural systems can be understood by looking at their interaction, in a bi-directional closed-loop, with their external environment. Moreover, the environment itself can be manipulated, and the changes in dynamic behavior resulting from changes in the environment provide useful information for understanding the neural systems themselves. These experimental paradigms allow manipulations of the neural system under study that once were only possible with simulations on detailed computational models. "Biological" closed-loop experiments (Figures 1B,C) have been performed at single neuron level, by interfacing artificial and actual neurons (Le Masson et al., 2002) at population level, by controlling the dynamic regime of neuronal populations (Wagenaar et al., 2005), and by investigating basic mechanisms of learning (Shahaf and Marom, 2001; Le Feber et al., 2010) and at the level of a "kind of whole organism," in experiments in which portions of nervous 
tissue are connected to artificial or virtual (Reger et al., 2000; DeMarse et al., 2001; Kositsky et al., 2009), or artificial/hybrid animals (Novellino et al., 2007; Tessadori et al., 2012). It was found that unidirectional (open-loop) periodic perturbation mode resulted in entrainment loss, while bidirectional (closedloop) mode is able to maintain the phase-locked entrainment (Jung et al., 2001). Closed-loop experiments are also relevant to the technology of neural interfaces (Mussa-Ivaldi and Miller, 2003; Nicolelis, 2003; Berger et al., 2011; Bonifazi et al., 2013). In fact, the latter implies the ability to monitor neural activity at population level in real-time and, conversely, to generate patterns of time- and space-varying stimuli, again in real-time.

These results prove that a connection between biological and artificial systems is possible creating a conditioning black box, in other words a translator that adapts the time-, space-, amplitude, and shape characteristics of electrical stimuli (Vassanelli and Mahmud, 2016). Clearly the more intrinsically neuromorphic a synthetic network is, the less complex this conditioning bottleneck will be. Hence Resistive Switching Devices (RSDs), that possess unique properties in this respect, well represent to date the most advanced condensed matter candidate for a direct coupling with living neuronal systems. Nevertheless interfacing living biological brains is still a though target to be achieved, we are experiencing the first recent promising results (Gupta et al., 2016a). Worth mentioning also the strong effort in finding soft solutions for electrodes featuring a complete matching (not yet announced) of mechanical properties with biological tissues (Rogers et al., 2016), or the many attempts of interfacing the peripheral nervous system for the control of neuroprostheses and hybrid bionic systems (Navarro et al., 2005).

\section{INTRINSIC NEUROMORPHIC PROPERTIES OF RSDs AND COUPLING WITH LIVING NEURONS}

The best prototype of an intrinsic neuromorphic system is perhaps the memristor, a promising solution for the beyondMoore era of nanoelectronics. In 1971, L. O. Chua predicted the existence of a fourth fundamental device (meaning that it cannot be realized using passive devices), which he called a "memristor," contraction of "memory resistor" (Chua, 1971). Since then, many RSDs were developed exploiting the properties of a plethora of nanoscale materials (Calzecchi-Onesti, 1884; Sawa, 2008; Strukov et al., 2008; Pershin and Di Ventra, 2011; Yang et al., 2013, 2016; Lin et al., 2014; Porro et al., 2015; Laurenti et al., 2017), even in liquid form (Chiolerio et al., 2016).

Currently we talk about RSDs in place of memristors, as the diverse mechanisms that enable device switching do not feature the ideal properties required by Chua's ideal bipole (Vonger and Xiangkang, 2015).

RSDs were proposed as neuromorphic emulators (Snider, 2008; Pershin et al., 2009). Crossbar RSDs network can potentially offer connectivity similar to neurons with RSDs working as synapses. Moreover the function density $\left(10^{10} / \mathrm{cm}^{2}\right)$ comparable to those of biological systems can be potentially obtained with advanced lithography approaches. Regarding neuromorphic functionalities that can be found in artificial systems, RSDs were shown to possess:

1. short term plasticity (STP);

2. long term plasticity (LTP);

3. spike-timing dependent plasticity (STDP);

4. spike-rate dependent plasticity (SRDP).

STP is the temporal potentiation of synaptic connections, lasting from seconds to minutes and then fading away with the ceasing of the stimulus. LTP is a more prolonged potentiation, lasting for years or even permanent. STDP is thought to be the most important feature of synaptic plasticity in biological brains. It implies that the synaptic weight varies according to the timing of the pre- and post-synaptic spikes. If a post-synapse is stimulated after a pre-synapse $(\Delta t<0)$, the synaptic weight is increased and produces a long-term effect (LTP). If the order is reversed $(\Delta t>0)$, the synaptic weight drops and there is a long term depression (LTD). Furthermore, the strength of synaptic plasticity is proportional to the pre-synaptic spiking rate (SRDP). (Jo et al., 2010; Li S. et al., 2013; Chen et al., 2014).

The first confirmation of STDP was obtained from Jo et al. (2010) using a metal/insulator/metal (MIM) RSD that works by migration of silver ions with the formation of conductive metallic filaments. Also Li S. et al. (2013) invoked the formation of metallic filaments in this case the matrix was a conductive polymer PEDOT-PSS. While the LTP is believed to be due to the growth of the silver filaments, the PEDOT-PSS is responsible for the STP, STDP, and SRDP (Figures 1D-G). The formation of metallic filaments is believed to be hindered by the elastic recovery of the polymer while a "high-rate stimulation with either a high strength or high frequency will enhance ion movement, suppress the elastic recovery and then result in the LTP."

The retention of RSDs based on metal oxides decays exponentially, Chang et al. compare the memory decay of tungsten oxide RSDs to human memory loss (Chang et al., 2011). Wang et al. worked on amorphous indium gadolinium zinc oxides (Wang et al., 2012) demonstrating essential synaptic functions including SRDP, STDP, LTP/STP, and "learningexperience" behavior. The STP decay is explained with the relaxation processes determined by the back-diffusion of oxygen ions. The LTP is enhanced through high-rate stimulation with short time interval and repetitive stimulation training demonstrating thus SRDP and STDP. Subramaniam et al. integrated two different devices in the same circuit: TFT nanoparticles were used to produce STP and SRDP by reacting to pre-synaptic spike signals while metal oxide RSDs based on hafnium oxide was used as memristive device to simulate LTP (Subramaniam et al., 2013). This kind of system is now very popular and synapses analogs were fabricated using different types of metal oxides RSDs as reported in Table $\mathbf{1}$.

Krzysteczko et al. demonstrate that MTJ (Magnetic Tunnelling Junction) RSDs intrinsically exhibit the features of biological synapses and neurons (Pershin and Di Ventra, 2008). Repeated treatment with voltage pulses led to a gradual, non-volatile change in MTJ resistance emulating LTP and LTD. Voltage-induced resistance variation is described by flux providing the scope for the emulation of STDP. Similar 
A

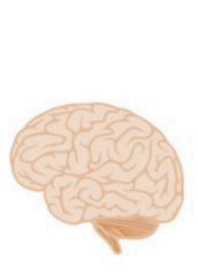

Neural Element

B

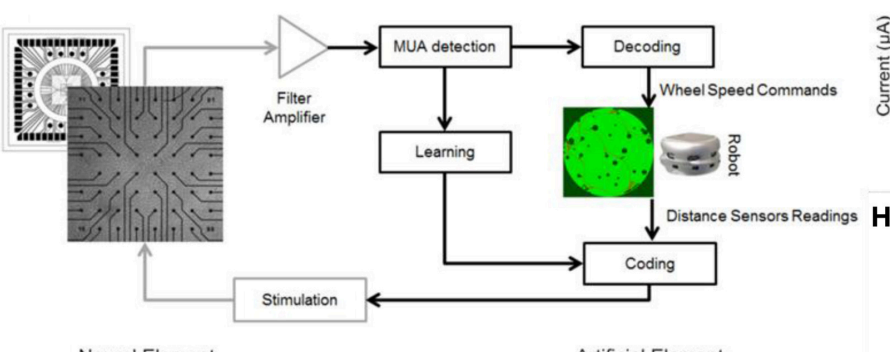

Neural Element

C

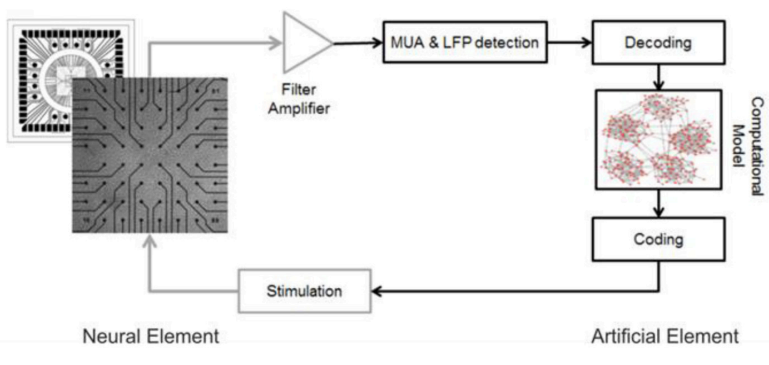

D

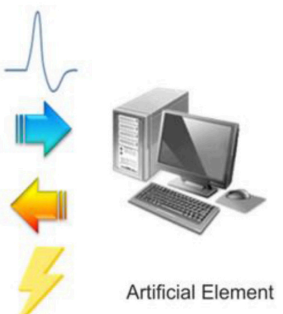

Artificial Element

Artificial Element

E
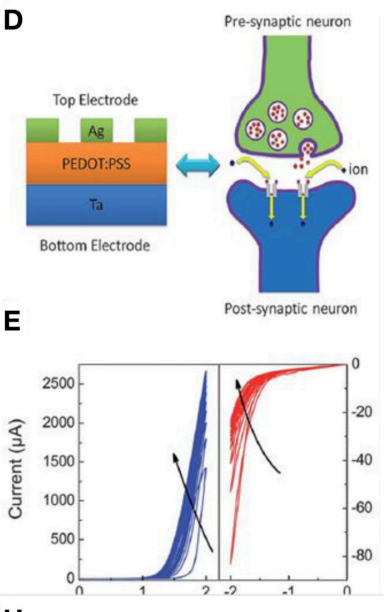

H

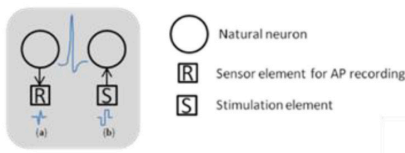

ANS

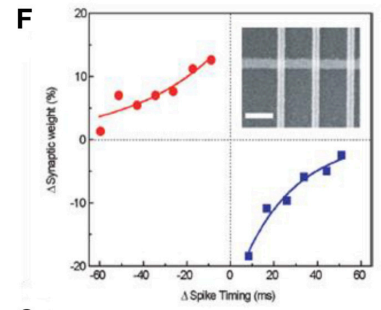

G

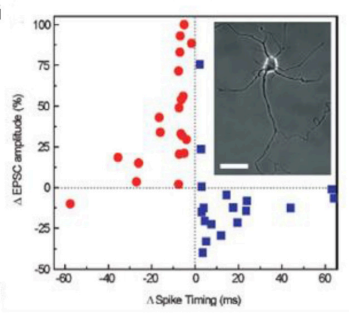

I

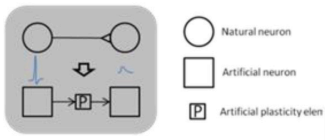

J

FIGURE 1 | Hybrid systems. (A) A cartoon of a neural hybrid system, which is typically composed by a neural element (the brain or a simplified model of it) and an artificial one (a computational device, in general). The two elements communicate through a bi-directional interaction realized through the acquisition of the "biosignals" from the neural element to the artificial one and, after data processing, a specific stimulation pattern is fed back to the neural element. (B) An example of neurorobotic system where a culture of dissociated neurons is able to bi-directional interact through a signal processing block (Multi-Unit-Activity, MUA, detection) with either a physical or a virtual robot (modified from Tessadori et al., 2012). (C) An example of bidirectional interaction between a biological network coupled to a MEA and a computational model of a neural network: this experimental framework can be exploited for medical applications such as innovative cognitive/brain prostheses (modified from Bonifazi et al., 2013). The communication between the artificial element and its neuronal counterpart is accomplished by the "Coding" and "Decoding" blocks in both panels (C,D). (D) Analogy between a multilayered hybrid memristor and a biological synapse: structural analogy in a Ag/PEDOT:PSS/Ta stack and biological synapse. (E) IV curves obtained after multiple consecutive scans. Panels (D,E) reprinted with permission from Li S. et al. (2013). (F) Comparison between synthetic and biological STDP measurements: synaptic weight as a function of the synchronization timing between pre and post-synaptic signals in a network of memristive devices. (G) STDP effect in living biological neurons. Panels (F,G) reprinted with permission from Jo et al. (2010). (H) Communication to real physical neurons established through micro -nanoelectronic components. (I) Memristive synapse: a physical plasticity component is developed to emulate natural synapse behavior. (L) Synaptors: signal transmission between artificial and natural neurons. Panels (H-L) adapted from http://www.rampproject.eu/project-objectives, last accessed May 26th 2016.

results were obtained from Wang et al. (2014) while this kind of RSD was used to build an on-chip pattern recognition of a multishaded grayscale image in a neural network circuit with multiple neurons (Kaneko et al., 2014).

The charge transferred under pulsed operation to an RSD compared with the amount exchanged by synapses is usually quite high. Also the electric potential of the spike is typically at least one order of magnitude higher. Yet, recent findings demonstrate that soft materials are able to set and reset in a narrow voltage range comprised between 50 and $100 \mathrm{mV}$ (Rajan et al., 2016) with On currents as low as $40 \mu \mathrm{A}$ and Off currents of $600 \mathrm{nA}$ (Rajan et al., 2017), that could easily be matched to induce living neurons through an impedance load connected in parallel. The main problem in coupling RSDs networks with living neurons is the higher range of power used to change RSD state, in the range of $250 \mu \mathrm{J}$ (Gupta et al., 2016b) and the low energy and power density of neurons. Indeed up to now there are two reports showing the first experimental realization of an hybrid network of living neuronal cells connected to a layer of artificial spiking neurons (Gater et al., 2014; Gupta et al., 2016a). Quite similar systems were shown to result in a multistate resistive switching, hence reducing the distance 
TABLE 1 | Resistive Switching Devices intrinsic neuromorphic properties in a comparison table, according to existing literature.

\begin{tabular}{|c|c|c|c|c|c|c|}
\hline Family & STP & LTP & STDP & SRDP & Type & References \\
\hline \multirow[t]{2}{*}{ Metal filament } & & & $x$ & & Array & Jo et al., 2010 \\
\hline & $x$ & $\mathrm{x}$ & $x$ & $\mathrm{x}$ & Single & Li S. et al., 2013 \\
\hline \multirow[t]{11}{*}{ Metal oxide } & $\mathrm{x}$ & $\mathrm{x}$ & & & Single & Chang et al., 2011 \\
\hline & $x$ & $x$ & $\mathrm{x}$ & $\mathrm{x}$ & Single & Wang et al., 2012 \\
\hline & & $x$ & $x$ & & Single & Williamson et al., 2013 \\
\hline & $\mathrm{x}$ & $\mathrm{x}$ & $\mathrm{x}$ & & Array & Subramaniam et al., 2013 \\
\hline & & & $x$ & $x$ & Single & He et al., 2014 \\
\hline & & $x$ & $x$ & & Single & Kim et al., 2015 \\
\hline & & & $x$ & & Array & Wang Y.-F. et al., 2015 \\
\hline & & & $x$ & & Single & Du et al., 2015 \\
\hline & & & $x$ & & Array & Wang Z. et al., 2015 \\
\hline & & & $x$ & & Array & Matveyev et al., 2016 \\
\hline & & & $x$ & & Array & Prezioso et al., 2016 \\
\hline \multirow[t]{3}{*}{ Spintronic } & & $\mathrm{x}$ & $\mathrm{x}$ & & Single & Krzysteczko et al., 2012 \\
\hline & & & $\mathrm{x}$ & & Single & Wang et al., 2014 \\
\hline & & & $x$ & & Array & Kaneko et al., 2014 \\
\hline \multirow[t]{5}{*}{ Chalcogenide } & $x$ & $x$ & $\mathrm{x}$ & & Array & Ohno et al., 2011 \\
\hline & $x$ & $\mathrm{x}$ & $\mathrm{X}$ & & Single & Nayak et al., 2012 \\
\hline & & & $x$ & & Array & Kuzum et al., 2012 \\
\hline & & & $x$ & & Single & Li Y. et al., 2013 \\
\hline & & & $x$ & $x$ & Single & Li et al., 2014 \\
\hline
\end{tabular}

between neuromorphic devices and biological brains (Sandouk et al., 2016). Closed-loop systems may be realized by modulating the compliance current given as input to the RSDs network using as template the action potential of the biological synapse network, realizing a point-to-point spatial equivalence between the two networks and obtaining a dynamically coupled pair of non-linear oscillator networks (Jung et al., 2001).

We conclude that it is natural to expect a wide integration between the biological and the synthetic worlds (Demin et al., 2015; Wu et al., 2015; Feali and Ahmadi, 2016). Funding and research efforts are currently experiencing a slow shift from the focus on neuromorphic engineering and an extrinsic paradigm of emulation of biological processes (to cite some, project CORONET http://cordis.europa.eu/project/rcn/97109_ en.html) belonging to FP7, EU), to more open frameworks where the use of RSDs could help in closing the loop (such as RAMP project, http://www.rampproject.eu, EU, Figures $1 \mathbf{H}-\mathrm{L}$ ). Referring to this, we read "Artificial neural networks in the form of software run on conventional von Neumann computers appear incomparable to the biological systems in terms of speed, energy efficiency, adaptability, and robustness. The challenge is to build a physical neural network where elements overcome this deficiency by merging data storage and processing into single electronic devices [...] we aim to create a new biohybrid architecture of tightly coupled natural and artificial neurons endowed with plasticity properties. [...] Adaptation properties of the artificial network will rely on memristive nanoelectronic devices with synaptic-like plasticity and on activity-dependent rearrangement of neuronal connectivity. As such, the biohybrid system will provide new and unique adaptive, self-organizing and evolving properties deriving from the fusion of natural and artificial neuronal elements into a new plastic entity" The RAMP project reports the specific target that this Perspective is addressing: the "Development of physical components with plastic properties based on nano-scale memristors in combination with CMOS circuitry emulating the function of a biological synapse." Nevertheless we see, once more, that CMOS emulators are required to achieve the so called Synaptor, a new biohybrid signal transmission unit that couples together one natural and one artificial neuron.

We may also imagine that the direct coupling between two perfectly compliant neuromorphic systems could lead to spontaneous adaptation, locking and growth of the networks, that will behave as a single super-network and exchange freely information.

\section{CONCLUSIONS}

After being theorized 40 years ago, it is only in the last decade that the fabrication of intrinsically neuromorphic devices was demonstrated. Following this milestone, in the latest years relevant efforts in the scientific community was directed toward the development of new materials for RSDs as well as theoretical algorithms for their use. One of the most promising applications of RSDs is in developing neuronal networks.

On the other hand in the recent years it was possible to grow neurons over artificial substrates and new methodologies for the activity recording allowed the study of signals in neuronal networks and direct interaction with bio-artificial circuits, with a specific care in the simultaneous recording of signals from a complex network of neurons, in place of a single and isolated cell.

Coupling RSDs with neuronal networks is still a distant objective. Nowadays we are at the edge of a new era where it will be possible to conceive and develop systems with reliable electrical interfaces between the brain and RSD-based neuronal networks, with the possibility of integrating them in wearable and comfortable devices. Neural prosthetics will be interfaced directly without any computer translation and used to fight serious neurological conditions resulting from disease, aging, or injury. And what about enhancing cognitive capacities of healthy people? The logical next step.

\section{AUTHOR CONTRIBUTIONS}

All authors gave substantial contribution to the development of this work equally, drafting and revising it critically; furthermore all Authors approved its final version for publication.

\section{FUNDING}

This work was fully funded by Fondazione Istituto Italiano di Tecnologia.

\section{ACKNOWLEDGMENTS}

We gratefully acknowledge Candido Fabrizio Pirri as Coordinator of the Center for Space Human Robotics. 


\section{REFERENCES}

Ariano, P., Baldelli, P., Carbone, E., Gilardino, A., Giudice, A. L., Lovisolo, D., et al (2005). Cellular adhesion and neuronal excitability on functionalised diamond surfaces. Diam. Relat. Mater. 14, 669-674. doi: 10.1016/j.diamond.2004. 11.021

Ariano, P., Lo Giudice, A., Marcantoni, A., Vittone, E., Carbone, E., and Lovisolo, D. (2009). A diamond-based biosensor for the recording of neuronal activity. Biosens. Bioelectron. 24, 2046-2050. doi: 10.1016/j.bios.2008.10.017

Bartol, T. M., Bromer, C., Kinney, J., Chirillo, M. A., Bourne, J. N., Harris, K. M., et al. (2016). Nanoconnectomic upper bound on the variability of synaptic plasticity. Elife 4:e10778. doi: 10.7554/eLife.10778

Berdondini, L., Imfeld, K., Maccione, A., Tedesco, M., Neukom, S., and KoudelkaHep, M., et al (2009). Active pixel sensor array for high spatio-temporal resolution electrophysiological recordings from single cell to large scale neuronal networks. Lab Chip 9, 2644-2651. doi: 10.1039/b907394a

Berger, T. W., Hampson, R. E., Song, D., Goonawardena, A., Marmarelis, V. Z., and Deadwyler, S. A. (2011). A cortical neural prosthesis for restoring and enhancing memory. J. Neural Eng. 8:046017. doi: 10.1088/1741-2560/8/4/046017

Biffi, E., Regalia, G., Menegon, A., Ferrigno, G., and Pedrocchi, A. (2013). The influence of neuronal density and maturation on network activity of hippocampal cell cultures: a methodological study. PLOS ONE 8:e83899. doi: 10.1371/journal.pone.0083899

Bisio, M., Bosca, A., Pasquale, V., Berdondini, L., and Chiappalone, M. (2014). Emergence of bursting activity in connected neuronal sub-populations. PLoS ONE 9:e107400. doi: 10.1371/journal.pone. 0107400

Bologna, L. L., Nieus, T., Tedesco, M., Chiappalone, M., Benfenati, F., and Martinoia, S. (2010). Low-frequency stimulation enhances burst activity in cortical cultures during development. Neuroscience 165, 692-704. doi: 10.1016/j.neuroscience.2009.11.018

Bonifazi, P., Difato, F., Massobrio, P., Breschi, G. L., Pasquale, V., Levi, T., et al (2013). In vitro large-scale experimental and theoretical studies for the realization of bi-directional brain-prostheses. Front. Neural Circuits 7:40. doi: $10.3389 /$ fncir.2013.00040

Bonifazi, P., Ruaro, M. E., and Torre, V. (2005). Statistical properties of information processing in neuronal networks. Eur. J. Neurosci. 22, 2953-2964. doi: $10.1111 / j .1460-9568.2005 .04464 . x$

Breslin, C., and O'Lenskie, A. (2001). Neuromorphic hardware databases for exploring structure-function relationships in the brain. Philos. Trans. R. Soc. Lond B Biol. Sci. 356, 1249-1258. doi: 10.1098/rstb.2001.0904

Brooks, R., Hassabis, D., Bray, D., and Shashua, A. (2012). Turing centenary: is the brain a good model for machine intelligence?. Nature 482, 462-463. doi: $10.1038 / 482462 \mathrm{a}$

Calzecchi-Onesti, T. (1884). Sulla conduttività elettrica delle limature metalliche. Il Nuovo Cimento 16, 58-64. doi: 10.1007/BF02737267

Chang, T., Jo, S. H., and Lu, W. (2011). Short-term memory to long-term memory transition in a nanoscale memristor. ACS Nano 5, 7669-7676. doi: $10.1021 / \mathrm{nn} 202983 \mathrm{n}$

Chen, Y., Liu, G., Wang, C., Zhang, W., Li, R. W., and Wang, L. (2014). Polymer memristor for information storage and neuromorphic applications. Mater. Horiz. 1, 489-506. doi: 10.1039/C4MH00067F

Chiappalone, M., Bove, M., Vato, A., Tedesco, M., and Martinoia, S. (2006). Dissociated cortical networks show spontaneously correlated activity patterns during in vitro development. Brain Res. 1093, 41-53. doi: 10.1016/j.brainres.2006.03.049

Chiappalone, M., Massobrio, P., and Martinoia, S. (2008). Network plasticity in cortical assemblies. Eur. J. Neurosci. 28, 221-237. doi: 10.1111/j.1460-9568.2008.06259.x

Chiappalone, M., Vato, A., Berdondini, L., Koudelka-Hep, M., and Martinoia, S. (2007). Network dynamics and synchronous activity in cultured cortical neurons. Int. J. Neural Syst. 17, 87-103. doi: 10.1142/S0129065707000968

Chiolerio, A., Roppolo, I., Bejtka, K., Asvarov, A., and Pirri, C. F. (2016). Resistive hysteresis in flexible nanocomposites and colloidal suspensions: interfacial Coupling Mechanism unveiled. RSC Adv. 6, 56661-56667. doi: 10.1039/C6RA10503C

Chua, L. O. (1971). Memristor-the missing circuit element. Circuit Theory IEEE Trans. 18, 507-519. doi: 10.1109/TCT.1971.1083337
DeMarse, T. B., Wagenaar, D. A., Blau, A. W., and Potter, S. M. (2001). The neurally controlled animat: biological brains acting with simulated bodies. Auton. Robots 11, 305-310. doi: 10.1023/A:1012407611130

Demin, V. A., Erokhin, V. V., Emelyanov, A. V., Battistoni, S., Baldi, G., and Iannotta, S., et al. (2015). Hardware elementary perceptron based on polyaniline memristive devices. Org. Electron. 25, 16-20. doi: 10.1016/j.orgel.2015.06.015

Du, N., Kiani, M., Mayr, C. G., You, T., Burger, D., Skorupa, I., et al. (2015). Single pairing spike-timing dependent plasticity in $\mathrm{BiFeO}_{3}$ memristors with a time window of $25 \mathrm{~ms}$ to $125 \mu \mathrm{s}$. Front. Neurosci. 9:227. doi: $10.3389 /$ fnins.2015.00227

Eytan, D., and Marom, S. (2006). Dynamics and effective topology underlying synchronization in networks of cortical neurons. J. Neurosci. 26, 8465-8476. doi: 10.1523/JNEUROSCI.1627-06.2006

Feali, M. S., and Ahmadi, A. (2016). Realistic hodgkin-huxley axons using stochastic behavior of memristors. Neural Process. Lett. 1-14. doi: 10.1007/s11063-016-9502-5

Frey, U., Egert, U., Heer, F., Hafizovic, S., and Hierlemann, A. (2009). Microelectronic system for high-resolution mapping of extracellular electric fields applied to brain slices. Biosens. Bioelectron. 24, 2191-2198. doi: 10.1016/j.bios.2008.11.028

Gater, D., Iqbal, A., Davey, J., and Gale, E. (2014). Connecting spiking neurons to a spiking memristor network changes the memristor dynamics. arXiv:14 02.4029 .

Gramowski, A., Schiffmann, D., and Gross, G. W. (2000). Quantification of acute neurotoxic effects of trimethyltin using neuronal networks cultured on microelectrode arrays. Neurotoxicology 21, 331-342.

Gross, G. W., Azzazy, H. M. E., Wu, M. C., and Rhodes, B. K. (1995). The use of neuronal networks on multielectrode arrays as biosensors. Biosens. Bioelectron. 10, 553-567. doi: 10.1016/0956-5663(95)96931-N

Gross, G. W., Rieske, E., Kreutzberg, G. W., and Meyer, A. (1977). A new fixed-array multi-microelectrode system designed for long-term monitoring of extracellular single unit neuronal activity in vitro. Neurosci. Lett. 6, 101-105. doi: 10.1016/0304-3940(77)90003-9

Gupta, I., Serb, A., Khiat, A., Zeitler, R., Vassanelli, S., and Prodromakis, T. (2016a). Real-time encoding and compression of neuronal spikes by metal-oxide memristors. Nat. Commun. 7, 1-16. doi: 10.1038/ncomms 12805

Gupta, I., Serb, A., Khiat, A., Zeitler, R., Vassanelli, S., and Prodromakis, T. (2016b). Sub $100 \mathrm{nW}$ volatile nano-metal-oxide memristor as synaptic-like encoder of neuronal spikes. arXiv:1611.09671. doi: 10.1038/ncomms12805

He, W., Huang, K., Ning, N., Ramanathan, K., Li, G., Jiang, Y., et al. (2014). Enabling an integrated rate-temporal learning scheme on memristor. Sci. Rep. 4:4755. doi: $10.1038 /$ srep04755

Hebb, D. A. (1949). The Organization of Behavior: A Neuropsychological Theory. New York, NY: John Wiley And Sons, Inc.

Heer, F., Hafizovic, S., Franks, W., Blau, A., Ziegler, C., and Hierlemann, A. (2006). CMOS microelectrode array for bidirectional interaction with neuronal networks. Solid State Circuits IEEE J. 41, 1620-1629. doi: 10.1109/JSSC.2006.873677

Jackson, A., Mavoori, J., and Fetz, E. E. (2006). Long-term motor cortex plasticity induced by an electronic neural implant. Nature 444, 56-60. doi: $10.1038 /$ nature 05226

Jimbo, Y., and Kawana, A. (1992). Electrical stimulation and recording from cultured neurons using a planar array. Bioelectrochem. Bioenerg. 40, 193-204. doi: 10.1016/0302-4598(92)80067-Q

Jo, S. H., Chang, T., Ebong, I., Bhadviya, B. B., Mazumder, P., and Lu, W. (2010). Nanoscale memristor device as synapse in neuromorphic systems. Nano Lett. 10, 1297-1301. doi: 10.1021/nl904092h

Jung, R., Brauer, E. J., and Abbas, J. J. (2001). Real-time interaction between a neuromorphic electronic circuit and the spinal cord. IEEE Trans. Neural Syst. Rehabilitation Eng. 9, 319-326. doi: 10.1109/7333.948461

Kaneko, Y., Nishitani, Y., and Ueda, M. (2014). Ferroelectric artificial synapses for recognition of a multishaded image. IEEE Trans. Electron. Devices 61, 2827-2833. doi: 10.1109/TED.2014.2331707

Kaul, R. A., Syed, N. I., and Fromherz, P. (2004). Neuron-semiconductor chip with chemical synapse between identified neurons. Phys. Rev. Lett. 92:038102. doi: 10.1103/PhysRevLett.92.038102 
Kim, S., Du, C., Sheridan, P., Ma, W., Choi, S., and Lu, W. D. (2015). Experimental demonstration of a second-order memristor and its ability to biorealistically implement synaptic plasticity. Nano Lett. 15, 2203-2211. doi: 10.1021/acs.nanolett.5b00697

Kositsky, M., Chiappalone, M., Alford, S. T., and Mussa-Ivaldi, S. (2009). Brainmachine interactions for assessing the dynamics of neural systems. Front. Neurorobot. 3:1. doi: 10.3389/neuro.12.001.2009

Krzysteczko, P., Münchenberger, J., Schäfers, M., Reiss, G., and Thomas, A. (2012). The memristive magnetic tunnel junction as a nanoscopic synapse-neuron system. Adv. Mater. 24, 762-766. doi: 10.1002/adma.201103723

Kuzum, D., Jeyasingh, R. G. D., Lee, B., and Wong, H.-S. P. (2012). Nanoelectronic programmable synapses based on phase change materials for brain-inspired computing. Nano Lett. 12, 2179-2186. doi: 10.1021/nl201040y

Laurenti, M., Porro, S., Pirri, C. F., Ricciardi, C., and Chiolerio, A. (2017). Zinc oxide thin films for memristive devices: a review. Crit. Rev. Solid State Mater. Sci. 42, 153-172. doi: 10.1080/10408436.2016.1192988

Le Feber, J., Stegenga, J., and Rutten, W. L. (2010). The effect of slow electrical stimuli to achieve learning in cultured networks of rat cortical neurons. PLoS ONE 5:e8871. doi: 10.1371/journal.pone.0008871

Le Masson, G., Renaud-Le Masson, S., Debay, D., and Bal, T. (2002). Feedback inhibition controls spike transfer in hybrid thalamic circuits. Nature 417, 854-858. doi: $10.1038 /$ nature 00825

Li, S., Zeng, F., Chen, C., Liu, H., Tang, G., Gao, S., et al. (2013). Synaptic plasticity and learning behaviours mimicked through Ag interface movement in an $\mathrm{Ag} /$ conducting polymer/Ta memristive system. J. Mater. Chem. C. 1, 5292-5298. doi: 10.1039/c3tc30575a

Li, Y., Zhong, Y., Xu, L., Zhang, J., Xu, X., Sun, H., et al. (2013). Ultrafast synaptic events in a chalcogenide memristor. Sci. Rep. 3:1619. doi: 10.1038/srep01619

Li, Y., Zhong, Y., Zhang, J., Xu, L., Wang, Q., Sun, H., et al. (2014). Activity-dependent synaptic plasticity of a chalcogenide electronic synapse for neuromorphic systems. Sci. Rep. 4:4906. doi: 10.1038/srep04906

Lin, W. P., Liu, S. J., Gong, T., Zhao, Q., and Huang, W. (2014). PolymerBased resistive memory materials and devices. Adv. Mater. 26, 570-606. doi: 10.1002/adma.201302637

Maeda, E., Robinson, H. P., and Kawana, A. (1995). The mechanisms of generation and propagation of synchronized bursting in developing networks of cortical neurons. J. Neurosci. 15, 6834-6845.

Maher, M., Pine, J., Wright, J., and Tai, Y. C. (1999). The neurochip: a new multielectrode device for stimulating and recording from cultured neurons. J. Neurosci. Methods 87, 45-56. doi: 10.1016/S0165-0270(98)00156-3

Marom, S., and Shahaf, G. (2002). Development, learning and memory in large random networks of cortical neurons: lessons beyond anatomy. Q. Rev. Biophys. 35, 63-87. doi: 10.1017/S0033583501003742

Massobrio, P., Tessadori, J., Chiappalone, M., and Ghirardi, M. (2015). In vitro studies of neuronal networks and synaptic plasticity in invertebrates and in mammals using multielectrode arrays. Neural Plast. 2015:196195. doi: $10.1155 / 2015 / 196195$

Matveyev, Y., Kirtaev, R., Fetisova, A., Zakharchenko, S., Negrov, D., and Zenkevich, A. (2016). Crossbar nanoscale hfo2-based electronic synapses. Nanoscale Res. Lett. 11, 147. doi: 10.1186/s11671-016-1360-6

Mavoori, J., Jackson, A., Diorio, C., and Fetz, E. (2005). An autonomous implantable computer for neural recording and stimulation in unrestrained primates. J. Neurosci. Methods 148, 71-77. doi: 10.1016/j.jneumeth.2005.04.017

Mussa-Ivaldi, F. A., and Miller, L. E. (2003). Brain-machine interfaces: computational demands and clinical needs meet basic neuroscience. Trends Neurosci. 26, 329-334. doi: 10.1016/S0166-2236(03)00121-8

Mussa-Ivaldi, S., Alford, S. T., Chiappalone, M., Fadiga, L., Karniel, A., Kositsky, M., et al (2010). New perspectives on the dialogue between brains and machines. Front. Neurosci. 3:8. doi: 10.3389/neuro.01.008.2010

Navarro, X., Krueger, T. B., Lago, N., Micera, S., Stieglitz, T., and Dario, P. (2005). A critical review of interfaces with the peripheral nervous system for the control of neuroprostheses and hybrid bionic systems. J. Peripher. Nerv. Syst. 10, 229-258. doi: 10.1111/j.1085-9489.2005.10303.x

Nayak, A., Ohno, T., Tsuruoka, T., Terabe, K., Hasegawa, T., Gimzewski, J. K., et al. (2012). Controlling the synaptic plasticity of a $\mathrm{Cu} 2 \mathrm{~S}$ gap-type atomic switch. Adv. Funct. Mater. 22, 3606-3613. doi: 10.1002/adfm.201200640

Nicolelis, M. A. (2003). Brain-machine interfaces to restore motor function and probe neural circuits. Nat. Rev. Neurosci. 4, 417-422. doi: 10.1038/nrn1105
Novellino, A., D’angelo, P., Cozzi, L., Chiappalone, M., Sanguineti, V., and Martinoia, S. (2007). Connecting neurons to a mobile robot: an in vitro bidirectional neural interface. Comput. Intell. Neurosci. 2007:12725. doi: $10.1155 / 2007 / 12725$

Ohno, T., Hasegawa, T., Tsuruoka, T., Terabe, K., Gimzewski, J. K., and Aono, M. (2011). Short-term plasticity and long-term potentiation mimicked in single inorganic synapses. Nat. Mater. 10, 591-595. doi: 10.1038/nmat3054

Pearce, T. M., and Williams, J. C. (2007). Microtechnology: meet neurobiology. Lab Chip 7, 30-40. doi: 10.1039/B612856B

Pershin, Y. V., and Di Ventra, M. (2008). Spin memristive systems: memory effects in semiconductor spintronics. Phys. Rev. B 78:113309. doi: 10.1103/PhysRevB.78.113309

Pershin, Y. V., and Di Ventra, M. (2011). Memory effects in complex materials and nanoscale systems. Adv. Phys. 60, 145-227. doi: 10.1080/00018732.2010. 544961

Pershin, Y. V., La Fontaine, S., and Di Ventra, M. (2009). Memristive model of amoeba learning. Phys. Rev. E 80:021926. doi: 10.1103/PhysRevE.80.021926

Porro, S., Accornero, E., Pirri, C. F., and Ricciardi, C. (2015). Memristive devices based on graphene oxide. Carbon 85, 383-396. doi: 10.1016/j.carbon.2015.01.011

Potter, S. M., and DeMarse, T. B. (2001). A new approach to neural cell culture for long-term studies. J. Neurosci. Methods 110, 17-24. doi: 10.1016/S0165-0270(01)00412-5

Prezioso, M., Merrikh Bayat, F., Likharev, K., and Strukov, D. (2016). Self-adaptive spike-time-dependent plasticity of metal-oxide memristors. Sci. Rep. 6:21331. doi: $10.1038 /$ srep21331

Rajan, K., Bocchini, S., Chiappone, A., Roppolo, I., Perrone, D., Bejtka, K., et al. (2017). Spin-coated silver nanocomposite resistive switching devices. Microelectron. Eng. 168, 27-31. doi: 10.1016/j.mee.2016.10.004

Rajan, K.; Chiappone, A., Perrone, D., Bocchini, S., Roppolo, I., Bejtka, K., et al. (2016). Ionic liquid-enhanced soft resistive switching devices. RSC Adv. 6, 94128-94138. doi: 10.1039/C6RA18668H

Reger, B. D., Fleming, K. M., Sanguineti, V., Alford, S., and Mussa-Ivaldi, F. A. (2000). Connecting brains to robots: an artificial body for studying the computational properties of neural tissues. Artif. Life 6, 307-324. doi: 10.1162/106454600300103656

Rogers, J. A., Ghaffari, R., and Dae-Hyeong, K. (ed.). (2016). Stretchable Bioelectronics for Medical Devices and Systems. Basel: Springer International Publishing.

Sandouk, E. J., Gimzewksi, J. K., and, Stieg, A. Z. (2016). Multistate resistive switching in silver nanoparticle films. Sci. Technol. Adv. Mater. 16:045004 doi: 10.1088/1468-6996/16/4/045004

Sawa, A. (2008). Resistive switching in transition metal oxides. Mater. Today 11, 28-36. doi: 10.1016/S1369-7021(08)70119-6

Shahaf, G., and Marom, S. (2001). Learning in networks of cortical neurons. J. Neurosci. 21, 8782-8788.

Snider, G. S. (2008). "Spike-timing-dependent learning in memristive nanodevices," in IEEE/ACM International Symposium on Nanoscale Architectures. Anaheim, CA, 85-92.

Stegenga, J., Le Feber, J., Marani, E., and Rutten, W. L. (2010). Phase-dependent effects of stimuli locked to oscillatory activity in cultured cortical networks. Biophys. J. 98, 2452-2458. doi: 10.1016/j.bpj.2010.02.026

Stoppa, M., and Chiolerio, A. (2014). Wearable electronics and smart textiles: a critical review. Sensors 14, 11957-11992. doi: 10.3390/s140711957

Strukov, D. B., Snider, G. S., Stewart, D. R., and Williams, R. S. (2008). The missing memristor found. Nature 453, 80-83. doi: 10.1038/nature06932

Subramaniam, A., Cantley, K. D., Bersuker, G., Gilmer, D. C., and Vogel, E. M. (2013). Spike-timing-dependent plasticity using biologically realistic action potentials and low-temperature materials. IEEE Trans. Nanotechnol. 12, 450-459. doi: 10.1109/TNANO.2013.2256366

Tessadori, J., Bisio, M., Martinoia, S., and Chiappalone, M. (2012). Modular neuronal assemblies embodied in a closed-loop environment: toward future integration of brains and machines. Front. Neural Circuits 6:99. doi: 10.3389/fncir.2012.00099

Thewes, R., Bertotti, G., Dodel, N., Keil, S., Schrýder, S., Boven, K. H., et al. (2016). "Neural tissue and brain interfacing CMOS devices - an introduction to state-of-the-art, current and future challenges," in 2016 IEEE International Symposium on Circuits And Systems (ISCAS) (Montreal), 1826-1829. 
Van Pelt, J., Corner, M. A., Wolters, P. S., Rutten, W. L. C., and Ramakers, G. J. A. (2004). Longterm stability and developmental changes in spontaneous network burst firing patterns in dissociated rat cerebral cortex cell cultures on multielectrode arrays. Neurosci. Lett. 361, 86-89. doi: 10.1016/j.neulet.2003.12.062

Vassanelli, S. (2014). "Multielectrode and multitransistor arrays for in vivo recording," in Nanotechnology and Neuroscience: Nano-Electronic, Photonic and Mechanical Neuronal Interfacing, eds M. D. Vittorio, L. Martiradonna, and J. Assad (New York, NY: Springer), 239-267.

Vassanelli, S., and Mahmud, M. (2016). Trends and Challenges in neuroengineering: toward "intelligent" neuroprostheses through brain"brain inspired systems" communication. Front. Neurosci. 10:438. doi: $10.3389 /$ fnins.2016.00438

Vassanelli, S., Mahmud, M., Girardi, S., and Maschietto, M. (2012). On the way to large-scale and high-resolution brain-chip interfacing. Cogn. Comput. 4, 71-81. doi: $10.1007 /$ s12559-011-9121-4

Venkatraman, S., Elkabany, K., Long, J. D., Yao, Y., and Carmena, J. M. (2009). A system for neural recording and closed-loop intracortical microstimulation in awake rodents. Biomed. Eng. IEEE Trans. 56, 15-22. doi: 10.1109/TBME.2008.2005944

Vonger, S., and Xiangkang, M. (2015). The missing memristor has not been found. Sci. Rep. 5:11657 doi: 10.1038/srep11657

Wagenaar, D. A., Madhavan, R., Pine, J., and Potter, S. M. (2005). Controlling bursting in cortical cultures with closed-loop multi-electrode stimulation. J. Neurosci. 25, 680-688. doi: 10.1523/JNEUROSCI.4209-04.2005

Wang, Y.-F., Lin, Y.-C., Wang, I.-T., Lin, T.-P., and Hou, T.-H. (2015). Characterization and modeling of nonfilamentary $\mathrm{Ta} / \mathrm{TaOx} / \mathrm{TiO} 2 / \mathrm{Ti}$ analog synaptic device. Sci. Rep. 5:10150. doi: 10.1038/srep10150

Wang, Z., Ambrogio, S., Balatti, S., and Ielmini, D. (2015). A 2-transistor/1resistor artificial synapse capable of communication and stochastic learning in neuromorphic systems. Front. Neurosci. 9:438. doi: 10.3389/fnins.2014. 00438

Wang, Z. Q., Xu, H. Y., Li, X. H., Yu, H., Liu, Y. C., and Zhu, X. J. (2012). Synaptic learning and memory functions achieved using oxygen ion migration/diffusion in an amorphous InGaZnO memristor. Adv. Funct. Mater. 22, 2759-2765. doi: 10.1002/adfm.201103148

Wang, Z., Zhao, W., Kang, W., Zhang, Y., Klein, J. O., Ravelosona, D., et al. (2014). Compact modelling of ferroelectric tunnel memristor and its use for neuromorphic simulation. Appl. Phys. Lett. 104:053505. doi: 10.1063/1.48 64270

Wiener, N. (1961). Cybernetics or Control and Communication in the Animal and the Machine, Vol. 25. Cambridge, MA: MIT Press.

Williamson, A., Schumann, L., Hiller, L., Klefenz, F., Hoerselmann, I., Husar, P., et al. (2013). Synaptic behavior and STDP of asymmetric nanoscale memristors in biohybrid systems. Nanoscale 5, 7297-7303. doi: 10.1039/c3nr0 $1834 \mathrm{~b}$

Wu, X., Saxena, V., and Zhu, K. (2015). "A CMOS spiking neuron for dense memristor-synapse connectivity for brain-inspired computing," in 2015 International Joint Conference on Neural Networks (IJCNN) (Killarney: IEEE).

Yang, J. J., Strukov, D. B., and Stewart, D. R. (2013). Memristive devices for computing. Nat. Nanotechnol. 8, 13-24. doi: 10.1038/nnano.2012.240

Yang, X., Wang, C., Shang, J., Zhang, C., Tan, H., Yi, X., et al. (2016). An organic terpyridyl-iron polymer based memristor for synaptic plasticity and learning behavior simulation. RSC Adv. 6, 25179-25184. doi: 10.1039/C6RA0 $2915 \mathrm{~A}$

Conflict of Interest Statement: The authors declare that the research was conducted in the absence of any commercial or financial relationships that could be construed as a potential conflict of interest.

Copyright (c) 2017 Chiolerio, Chiappalone, Ariano and Bocchini. This is an openaccess article distributed under the terms of the Creative Commons Attribution License (CC BY). The use, distribution or reproduction in other forums is permitted, provided the original author(s) or licensor are credited and that the original publication in this journal is cited, in accordance with accepted academic practice. No use, distribution or reproduction is permitted which does not comply with these terms. 